\title{
Structure-based and ligand-based virtual screening of novel methyltransferase inhibitors of the dengue virus
}

\author{
See Ven Lim', Mohd Basyaruddin A Rahman ${ }^{1,2}$, Bimo A Tejo ${ }^{1 *}$ \\ From Asia Pacific Bioinformatics Network (APBioNet) Tenth International Conference on Bioinformatics - First \\ ISCB Asia Joint Conference 2011 (InCoB2011/ISCB-Asia 2011) \\ Kuala Lumpur, Malaysia. 30 November - 2 December 2011
}

\begin{abstract}
Background: The dengue virus is the most significant arthropod-borne human pathogen, and an increasing number of cases have been reported over the last few decades. Currently neither vaccines nor drugs against the dengue virus are available. NS5 methyltransferase (MTase), which is located on the surface of the dengue virus and assists in viral attachment to the host cell, is a promising antiviral target. In order to search for novel inhibitors of NS5 MTase, we performed a computer-aided virtual screening of more than 5 million commercially available chemical compounds using two approaches: i) structure-based screening using the crystal structure of NS5 MTase and ii) ligand-based screening using active ligands of NS5 MTase. Structure-based screening was performed using the LIDAEUS (Llgand Discovery At Edinburgh UniverSity) program. The ligand-based screening was carried out using the EDULISS (EDinburgh University Llgand Selection System) program.
\end{abstract}

Results: The selection of potential inhibitors of dengue NS5 MTase was based on two criteria: the compounds must bind to NS5 MTase with a higher affinity than that of active NS5 MTase ligands, such as ribavirin triphosphate (RTP) and S-adenosyl-L-homocysteine (SAH); and the compounds must interact with residues that are catalytically important for the function of NS5 MTase. We found several compounds that bind strongly to the RNA cap site and the S-adenosyl-L-methionine (SAM) binding site of NS5 MTase with better binding affinities than that of RTP and SAH. We analyzed the mode of binding for each compound to its binding site, and our results suggest that all compounds bind to their respective binding sites by interacting with, and thus blocking, residues that are vital for maintaining the catalytic activity of NS5 MTase.

Conclusions: We discovered several potential compounds that are active against dengue virus NS5 MTase through virtual screening using structure-based and ligand-based methods. These compounds were predicted to bind into the SAM binding site and the RNA cap site with higher affinities than SAH and RTP. These compounds are commercially available and can be purchased for further biological activity tests.

\section{Background}

Dengue fever is an ancient disease that was first recorded in a Chinese encyclopedia of diseases and symptoms published during the Chin Dynasty (265 to 420 AD) [1]. Referred to as "water poison" in ancient Chinese medical

\footnotetext{
* Correspondence: bimotejo@science.upm.edu.my

'Department of Chemistry, Faculty of Science, Universiti Putra Malaysia, 43400 UPM Serdang, Malaysia

Full list of author information is available at the end of the article
}

literatures due to its association with flying insects bred in clean water, modern science has now confirmed that dengue fever is a viral disease transmitted between human hosts by Aedes mosquitoes, particularly Aedes aegypti. The ability of Aedes aegypti to adapt well to urban living environments plays a significant role in the outbreak of dengue fever [2]. Currently, dengue fever is the most important tropical infectious disease after malaria, and more than 100 countries have reported 
infections, especially countries in tropical and subtropical regions [3]. An estimated 100 million cases of dengue fever occur annually. Of these, 500,000 cases require hospitalization, and 25,000 are fatal $[1,4]$, particularly in developing and underdeveloped countries where access to healthcare facilities is limited.

In general, the dengue virus (DV) is a plus-strand RNA virus of the Flavivirus genus of the Flaviviridae family [5]. The DV has an approximately $50 \mathrm{~nm}$ envelope and contains a $10.7 \mathrm{~kb}$ single strand RNA that is translated into a single polyprotein followed by co-translational cleavage into 10 mature proteins. These 10 mature proteins consist of three structural proteins (capsid (c), premembrane (prM), envelope (E)) and seven nonstructural proteins (NS1, NS2A, NS2B, NS3, NS4A, NS4B, and NS5) (Figure 1) [6]. The nonstructural proteins are involved in evading innate immune responses, virion assembly, and the replication of the genome. The structural proteins play a role in the formation of the viral particle [6-8]. To date, the enzymatic activities of NS3 and NS5 are the best characterized among the non-structural DV proteins. Based on a number of studies, the methyltransferase (MTase) domain of the DV non-structural protein NS5 (NS5 MTase) is thought to be a promising antiviral target [9-12].
The stability of dengue viral mRNA and the efficient translation are closely related to its cap structure. The $5^{\prime}$ end of the dengue RNA has a type 1 cap structure $\left(\mathrm{me}^{7}\right.$ GpppA-me ${ }^{2}$ ), whose formation requires NS3 and NS5 enzymatic reactions [13]. NS5 MTase methylates the guanosine cap at the N7 position by transferring a methyl group from $S$-adenosyl-L-methionine (SAM) followed by methylation of the $2^{\prime}-\mathrm{OH}$ position of the first ribose nucleotide [14]. A side product, $S$-adenosyl-L-homocysteine $(\mathrm{SAH})$, is produced from N7 and 2'-O methylations [15]. Although MTase exhibits two distinct methylations, it has only single SAM binding site in its crystal structure [16]. It has been suggested that the substrate GpppA-RNA must be re-positioned to accept the N7 and 2'-O methyl groups from SAM during the two methylation reactions. There are two binding sites connected by a Y-shaped cleft. The first site is the SAM (methyl donor) binding site, and the second site is a shallow pocket of the RNA cap site [17].

NS5 MTase has a globular shape with a seven-stranded central $\beta$-sheet surrounded by six helices (Figure 2a). The crystal structure of NS5 MTase with SAH and a nucleoside analogue, ribavirin triphosphate (RTP), is shown in Figure $2 \mathrm{~b}$. Based on the crystal structure, there are two complexes assigned to two binding sites. One site is for

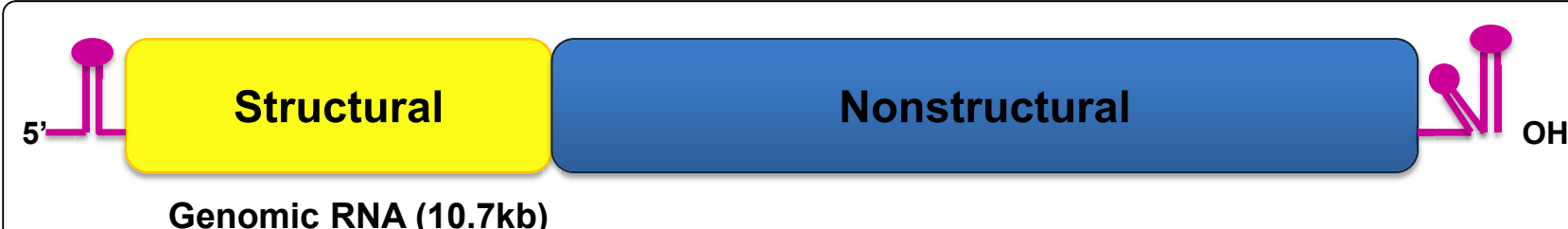

Genomic RNA (10.7kb)
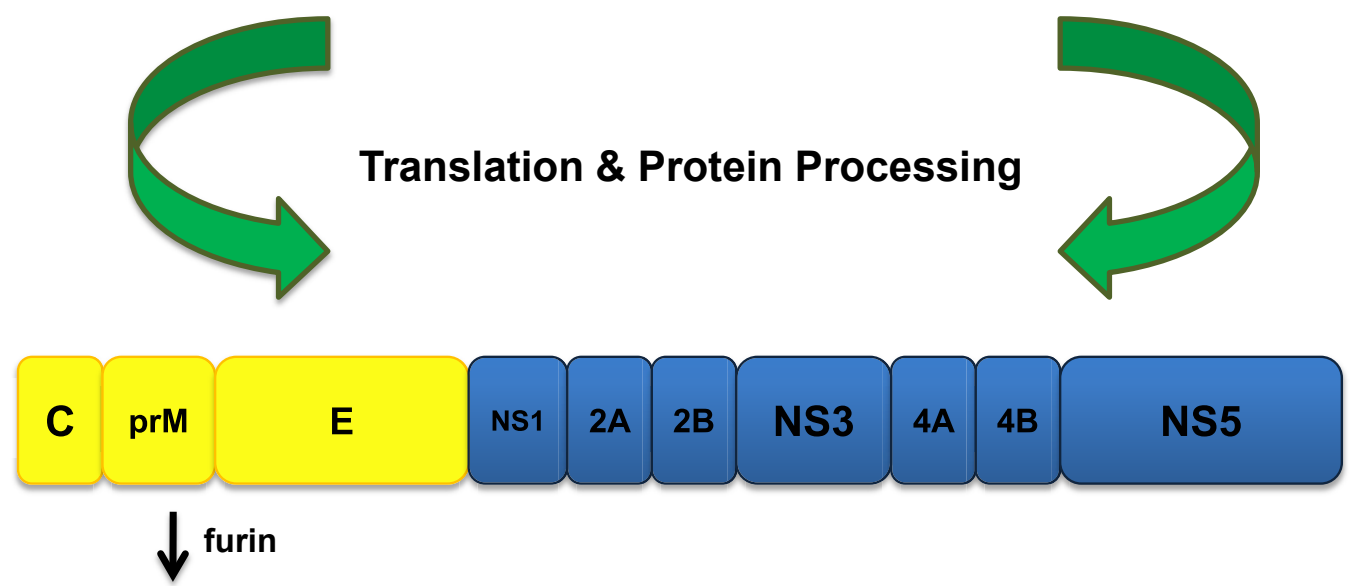

M

Viral Proteins

Figure 1 Schematic figure of Flavivirus RNA and its translation into proteins involved in the DV lifecycle. 


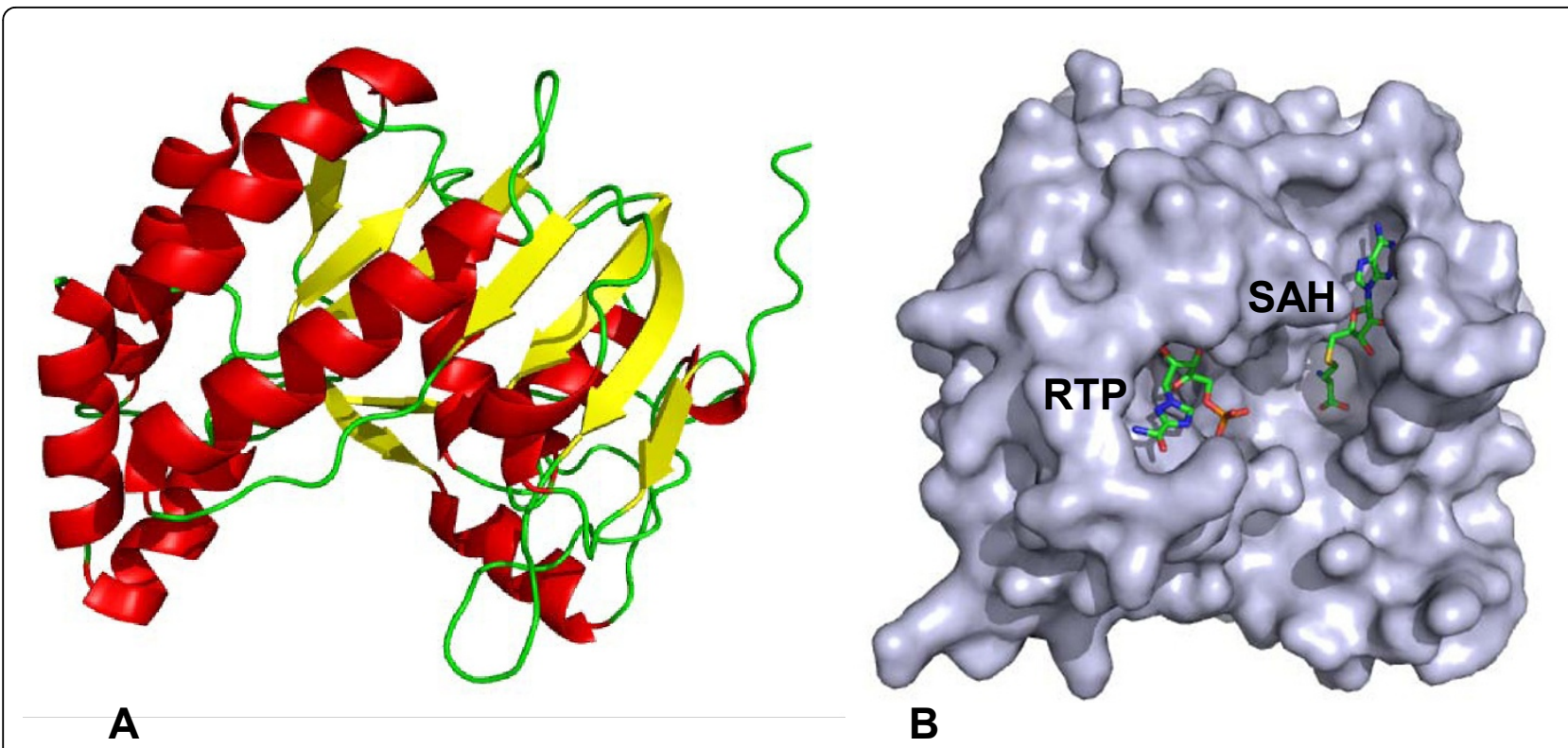

Figure 2 Structure of dengue NS5 MTase [PDB:1R6A]. (a) Ribbon representation of NS5 MTase; $\alpha$-helix is illustrated in red and $\beta$-sheet is illustrated in yellow. (b) Surface representation of NS5 MTase with RTP and SAH inside the RNA cap site and SAM binding site, respectively.

SAH and the methyl donor, SAM, and the second site is situated on an N-terminal subdomain for guanosine-5'-triphosphate (GTP) analogues [18].

Currently, there are no vaccines or drugs to inhibit DV replication and transmission. The presence of four serotypes of the DV (DV1 - DV4) has hampered the efforts to develop effective drugs or vaccines against the DV [19]. Infection with one DV serotype will result in lifelong immunity to that serotype; however, sequential infection by other serotypes of the DV will result more severe symptoms. Dengue hemorrhagic fever (DHF) and dengue shock syndrome (DSS) typically occur through antibody-mediated disease enhancement (ADE), either from previous DV infection [20] or from vaccine-induced ADE [21]. The unique properties of the DV make the design and discovery of effective vaccines and drugs against the DV extremely challenging.

In this work, we performed a computer-aided virtual screen of more than 5 million commercially available chemical compounds to find potential inhibitors of NS5 MTase. We utilized two different approaches: i) structurebased virtual screening using the crystal structure of NS5 MTAse [PDB:1R6A] and ii) ligand-based virtual screening using active ligands of NS5 MTase (ribavirin and SAH). Structure-based compound screening was performed using the LIDAEUS (LIgand Discovery At Edinburgh UniverSity) program [22], which maps the active site of the receptor and screens it against approximately 5 million commercially available chemical compounds in its database. Meanwhile, ligand-based compound screening was carried out using the EDULISS (EDinburgh University
LIgand Selection System) program [23], which utilizes known natural substrates of NS5 MTase to find structurally similar compounds from its database. We discovered several compounds that fit into the two binding sites of NS5 MTase with a higher binding affinity than its active ligands. These compounds can be further tested to confirm their biological activities against the DV.

\section{Results}

In this work, we employed two different approaches to virtually screen potential inhibitors against dengue virus NS5 MTase. A structure-based virtual screen was performed using LIDAEUS to generate a map based on the NS5 MTase binding site. This map was used to screen approximately 5 million compounds in the LIDAEUS database for those that fit into the binding site map. Meanwhile, a ligand-based virtual screen was done using the UFSRAT (Ultra Fast Shape Recognition with Atom Types) algorithm implemented in EDULISS to search for compounds that are structurally similar to the two active ligands of NS5 MTase, RTP and SAH.

We then selected the top 500 highest ranked compounds from the EDULISS and LIDAEUS screenings for further molecular docking experiments using AutoDock Vina. Even though LIDAEUS analyzed the initial docking of millions of compounds in its database to methyltransferase, it uses a course-grained docking tool that is essentially rigid body docking (rigid ligand and rigid protein). This is the only approach available for working with large datasets. LIDAEUS tries to get around the rigid-rigid limitation by representing multiple conformers of a small 
molecule within the dataset. Once LIDAEUS has identified the top small molecules, more accurate analysis/docking trials were performed using AutoDock Vina for flexible ligand docking. This is much more computationally expensive than the rigid-rigid docking carried out with LIDAEUS. Furthermore, EDULISS does not have the same automated docking functionality as LIDAEUS. AutoDock Vina was run to rank the proposed compounds from the EDULISS screen based on their binding affinities.

In total, we docked 2000 unique compounds from the LIDAEUS screen for molecules that potentially bind to SAM binding site (500 compounds) and RNA cap site (500 compounds) and from the EDULISS screen using RTP (500 compounds) and SAH (500 compounds) as templates. Binding affinity calculations for all 2000 compounds were carried out using an algorithm implemented in AutoDock Vina. We identified 40 compounds with the highest binding affinities to the SAM and RNA cap sites (Table 1) that have stronger binding to their respective binding sites than RTP and SAH. The rational basis of selecting compounds with binding affinities higher than that of RTP and SAH is that only compounds that bind to NS5 MTase with a higher affinity than its natural substrates can be developed as potential inhibitors. Only the compounds that are ranked first for the SAM and RNA cap sites will be discussed in detailed.

\section{Validation of the docking}

In molecular docking, the size and center of the coordinates of the grid box need to be validated in order to ensure that ligands bind to the binding pocket in the correct conformation. In this work, docking validation was performed by redocking co-crystallized RTP and SAH into their respective binding sites. We found that the binding conformations of redocked RTP and SAH reproduced the binding modes of the co-crystallized ligands with binding affinities of $-5.6 \mathrm{kcal} / \mathrm{mol}$ and $-7.3 \mathrm{kcal} / \mathrm{mol}$ for RTP and SAH, respectively (Figure 3).

The RNA cap site of NS5 MTase is an open and shallow pocket. There are three polar interactions formed between RTP and the RNA cap site (Figure 3a). The backbone oxygen of Leu20 forms a hydrogen bond with the N4 nitrogen of RTP, and the backbone oxygen of Asn18 forms a hydrogen bond with the N9 nitrogen. Both N4 and N9 are located on a pseudobase moiety of RTP, which mimics the interaction between the guanine moiety of GDPMP (a GTP analogue) and NS5 MTase [15]. The backbone nitrogen of Lys 29 forms a hydrogen bond with the O1P oxygen of RTP that further stabilizes RTP binding. A hydrophobic interaction between the pseudobase moiety of RTP and Leu17 of the RNA cap site was observed. The aromatic ring of Phe25 forms an aromatic stacking interaction with the pseudobase ring of RTP (Figure 3a).
The SAM binding pocket is thought to be more closed than the RNA cap site. The backbone oxygen of Ser56 and the nitrogen of Gly86 interact with the SAH molecule by forming hydrogen bonds with the carboxylic atoms of the homocysteine moiety of SAH. The carbonyl atom of the Asp146 side chain also forms a hydrogen bond with the amino group of the homocysteine moiety of SAH. In addition, there were four hydrophobic interactions between the hydrophobic side chains of Thr104, Lys105, Val132 and Ile147 in the SAM binding pocket with the adenosine moiety of the SAH molecule (Figure $3 \mathrm{~b}$ ).

Dong and colleagues [24] performed a biochemical and genetic characterization of the dengue virus NS5 MTase and identified important interactions between SAH and the surrounding residues of the SAM binding site. Asp131 interacts with the amino group of the adenosine moiety of SAH, while a hydrogen bond exists between the side chain of His110 and 2'-OH of the ribose moiety. Four residues (His110, Lys105, Asp131 and Ile147) were found to play an important role in N7 and 2- $\mathrm{O}^{\prime}$ methylations. That report emphasized the importance of the SAM binding site for both methylations. There are slight differences between our result and the result of Dong et al. in terms of the amino acids that are involved in the interaction between SAH and the SAM binding site. We identified three hydrogen bonds formed between SAH and Ser56, Gly86 and Asp146, which are not identified in Dong's work. However, our results confirm that Lys105 and Ile147 form hydrophobic interactions with SAH.

\section{Inhibitors bound to RNA cap site}

We performed molecular docking on 500 compounds obtained from the ligand-based EDULISS screen. These 500 compounds were ranked based on their binding affinities. The top ten compounds with binding affinites for the RNA cap site that are more negative than that of RTP are listed in Table 1. The two compounds that showed the strongest affinites for the RNA cap site of NS5 MTase were SPH1-103-799 and SPH1-101-102, each with a binding affinity of $-7.8 \mathrm{kcal} / \mathrm{mol}$. Meanwhile, 500 compounds from the structure-based LIDAEUS screen were also docked into the RNA cap site. The top 10 compounds with more negative binding affinities than that of RTP, indicating stronger binding, are listed in Table 1 . The compound with the highest affinity discovered through the LIDAEUS screen was 28SPH1-115-917, with a binding affinity of $-7.9 \mathrm{kcal} / \mathrm{mol}$.

Compound SPH1-103-799 (Figure 4a) forms three hydrogen bonds between oxygen $\mathrm{O} 35$ of the carbonyl group and the backbone nitrogen of Lys22, oxygen O36 of the hydroxyl group and the backbone oxygen of Leu20, and oxygen O30 of the hydroxyl group and the backbone oxygen of Asn18. Hydrophobic interactions occur between the aromatic rings of SPH1-103-799 and 
Table 1 Binding affinity and predicted binding pocket of docked compounds

\begin{tabular}{|c|c|c|c|}
\hline Compound ID & Virtual screening & Binding pocket & Binding affinity $(\mathrm{kcal} / \mathrm{mol})$ \\
\hline SPH1-103-799 & EDULISS & RNA cap & -7.8 \\
\hline SPH1-101-102 & EDULISS & RNA cap & -7.8 \\
\hline SPH1-027-074 & EDULISS & RNA cap & -7.7 \\
\hline SPH1-014-180 & EDULISS & RNA cap & -7.6 \\
\hline SPH1-047-692 & EDULISS & RNA cap & -7.6 \\
\hline SPH1-000-259 & EDULISS & RNA cap & -7.6 \\
\hline SPH1-013-274 & EDULISS & RNA cap & -7.5 \\
\hline SPH1-013-272 & EDULISS & RNA cap & -7.5 \\
\hline SPH1-013-273 & EDULISS & RNA cap & -7.5 \\
\hline SPH1-013-271 & EDULISS & RNA cap & -7.5 \\
\hline 28SPH1-115-917 & LIDAEUS & RNA cap & -7.9 \\
\hline $35 \mathrm{SPH} 1-021-288$ & LIDAEUS & RNA cap & -7.8 \\
\hline 28SPH1-185-015 & LIDAEUS & RNA cap & -7.8 \\
\hline 28SPH1-149-718 & LIDAEUS & RNA cap & -7.6 \\
\hline 28SPH1-026-800 & LIDAEUS & RNA cap & -7.6 \\
\hline 28SPH1-024-902 & LIDAEUS & RNA cap & -7.5 \\
\hline 28SPH1-081-432 & LIDAEUS & RNA cap & -7.5 \\
\hline 29SPH1-063-033 & LIDAEUS & RNA cap & -7.3 \\
\hline 28SPH1-024-902 & LIDAEUS & RNA cap & -7.3 \\
\hline 28SPH1-348-781 & LIDAEUS & RNA cap & -7.3 \\
\hline SPH1-007-088 & EDULISS & SAM & -11.4 \\
\hline SPH1-111-460 & EDULISS & SAM & -11.4 \\
\hline SPH1-177-492 & EDULISS & SAM & -11.0 \\
\hline SPH1-020-782 & EDULISS & SAM & -10.6 \\
\hline SPH1-016-182 & EDULISS & SAM & -10.5 \\
\hline SPH1-159-983 & EDULISS & SAM & -10.5 \\
\hline SPH1-129-393 & EDULISS & SAM & -10.4 \\
\hline SPH1-031-492 & EDULISS & SAM & -10.3 \\
\hline SPH1-107-521 & EDULISS & SAM & -10.3 \\
\hline SPH1-297-578 & EDULISS & SAM & -10.3 \\
\hline 25SPH1-103-433 & LIDAEUS & SAM & -10.0 \\
\hline 25SPH1-103-428 & LIDAEUS & SAM & -9.9 \\
\hline 42SPH1-001-864 & LIDAEUS & SAM & -9.8 \\
\hline 25SPH1-103-428 & LIDAEUS & SAM & -9.8 \\
\hline $42 \mathrm{SPH} 1-001-481$ & LIDAEUS & SAM & -9.5 \\
\hline 42SPH1-001-925 & LIDAEUS & SAM & -9.5 \\
\hline $25 \mathrm{SPH} 1-108-370$ & LIDAEUS & SAM & -9.3 \\
\hline 42SPH1-013-393 & LIDAEUS & SAM & -9.2 \\
\hline $25 \mathrm{SPH} 1-102-225$ & LIDAEUS & SAM & -9.1 \\
\hline 25SPH1-102-901 & LIDAEUS & SAM & -8.2 \\
\hline
\end{tabular}

Leu17, Phe25, Ser150, Pro152, and Ser214. The second potential inhibitor, compound SPH1-101-102 (Figure $4 \mathrm{~b})$, also forms three hydrogen bonds between the oxygen $\mathrm{O} 21$ of the carbonyl group with the backbone nitrogen of Lys14, oxygen $\mathrm{O} 22$ with the side chain oxygen of Ser150, and oxygen O13 with the side chain nitrogen of Lys22. In contrast with compound SPH1-103-799, only one residue is involved in hydrophobic interaction between SPH1-101-102 and the RNA cap site at Phe25. Meanwhile, compound 28SPH1-115-917 (Figure 4c) forms a hydrogen bond between oxygen O18 of the carbonyl group and the side chain oxygen of Ser150. Likewise, the side chain nitrogen atom of Lys 22 creates a hydrogen bond with oxygen $\mathrm{O} 5$. Two hydrophobic interactions are formed between the aromatic rings of 28SPH1-115-917 and Phe25 and Pro152.

Benarroch et al. [18], who studied the binding of RTP with the RNA cap site of NS5 MTase, suggested a list of residues that are significantly important for ligand binding: Lys14, Leu17, Asn18, Leu20, Phe25, Lys29, Ser150, Ser151, 


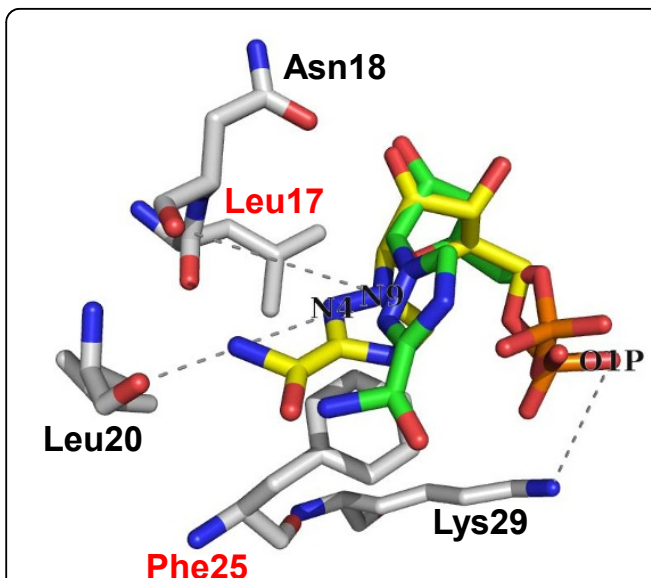

A

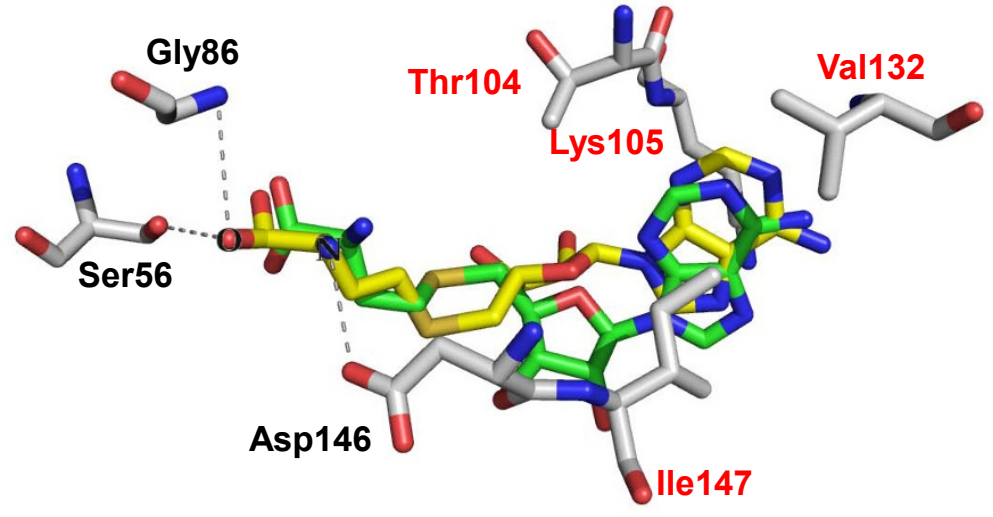

B

Figure 3 Redocking of RTP and SAH into RNA cap site and SAM binding site. (a) Crystallized conformation of RTP is shown in yellow carbons. The best redocked pose of RTP is shown in green carbons. (b) Crystallized conformation of SAH is shown in yellow carbons. The best redocked conformation of SAH is shown in green carbons. Residues with hydrophobic contacts with RTP and SAH are labeled in red and hydrogen bonds are drawn as dashed lines

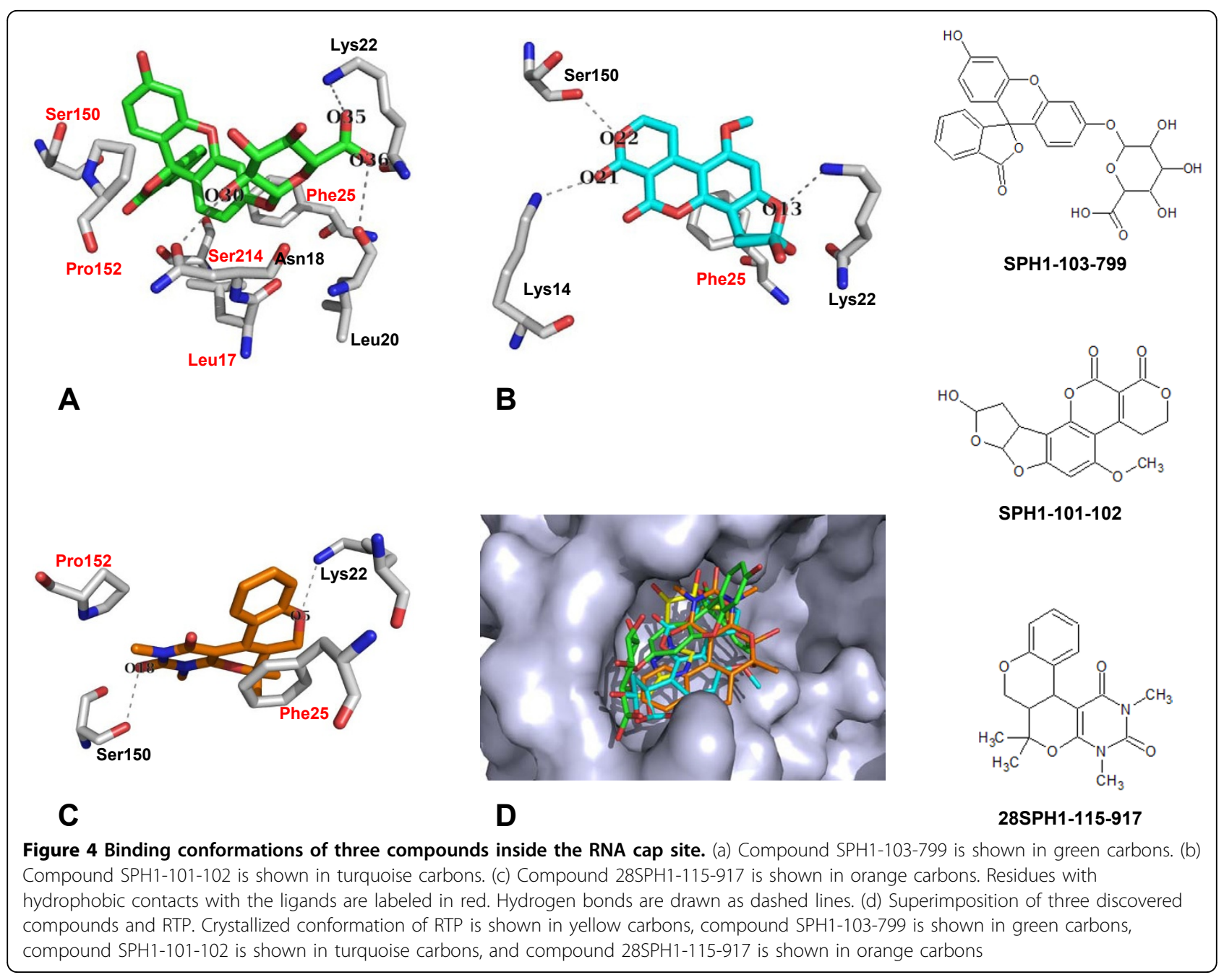


Pro152, Glu408, and Gly409. Our results show that compound SPH1-103-799 binds to the RNA cap site by forming interactions with eight residues: Leu17, Asn18, Leu20, Lys22, Phe25, Ser150, Pro152, and Ser214. These residues, with the exception of Lys22 and Ser214, interact with RTP in the crystal structure of NS5 MTase. Compounds SPH1101-102 and 28SPH1-115-917 have fewer interactions with the RNA cap site. Only four residues are involved in the binding of these two compounds. Even though compounds SPH1-101-102 and 28SPH1-115-917 have fewer interactions with the RNA cap site compared to RTP, these compounds show stronger binding to the RNA cap site than RTP. There are several possible explanations for this effect: i) compound SPH1-101-102 creates three hydrogen bonds with the RNA cap site at different parts of the ligand, thus distributing the strength of the binding all over the ligand molecule; ii) compound 28SPH1-115917 forms an aromatic stacking interaction with Phe25 that mimics the interaction of the pseudobase moiety of
RTP with Phe25, and the ligand binding is strengthened by two hydrogen bonds with Lys22 and Ser 150 .

\section{Inhibitors bound to SAM binding site}

After 500 compounds obtained from the ligand-based EDULISS screen were docked into the SAM binding site, 10 compounds with higher binding affinities for NS5 MTase than that of SAH were tabulated (Table 1). The two compounds with the strongest affinity for the SAM binding site were SPH1-007-088 and SPH1-111-460, each of which had a binding affinity of $-11.4 \mathrm{kcal} / \mathrm{mol}$. Meanwhile, 500 compounds obtained from the structurebased LIDAEUS screen were also docked into the SAM binding site, and the 10 best compounds are listed in Table 1 . The best binder was compound 25SPH1-103$433(-10.0 \mathrm{kcal} / \mathrm{mol})$.

Compound SPH1-007-088 (Figure 5a) forms two hydrogen bonds and several hydrophobic interactions with residues inside the SAM binding site. Two hydrogen

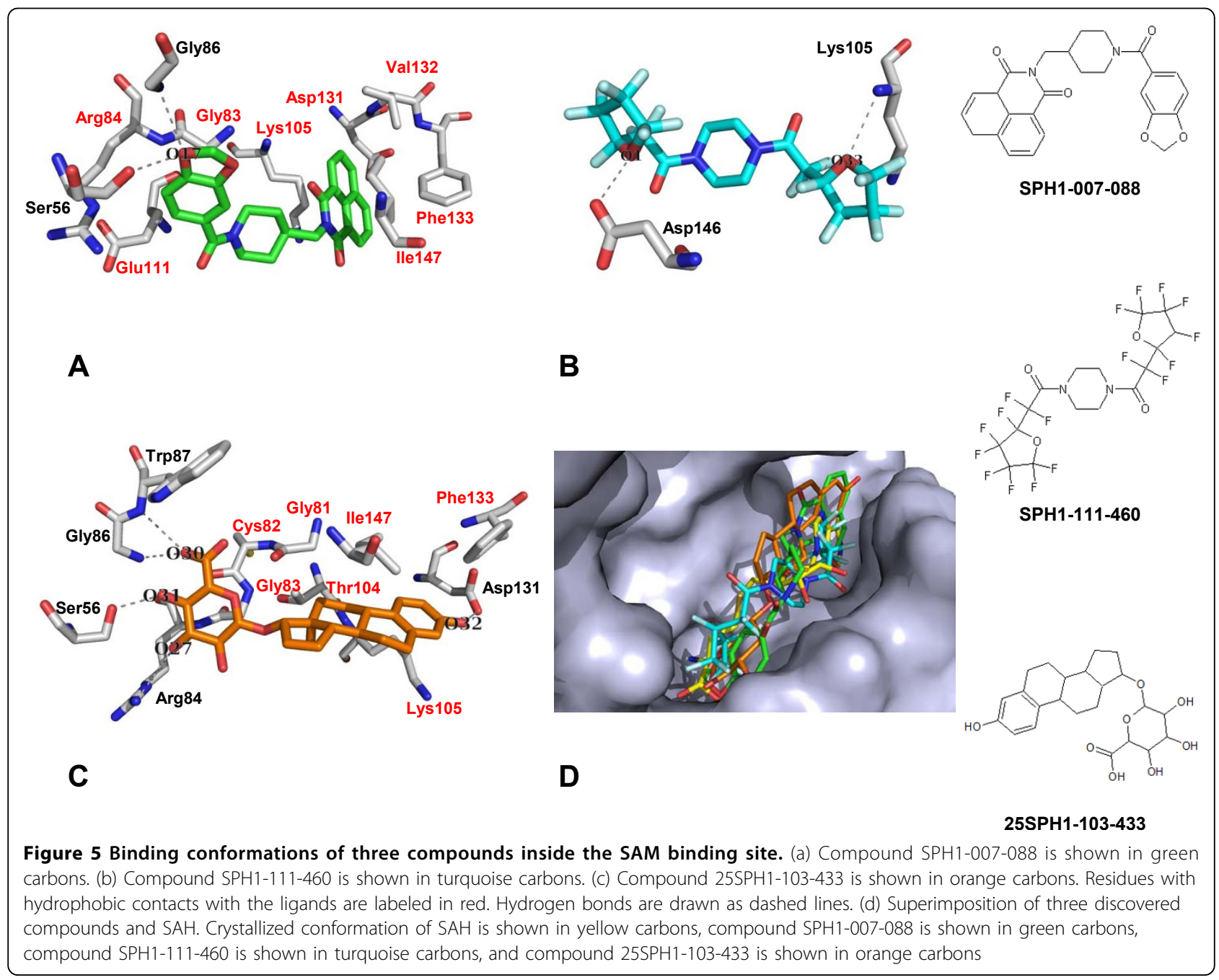


bonds are formed between the side chain oxygen of Ser56 and the backbone nitrogen of Gly86 with oxygen O17. In addition, several hydrophobic interactions are formed between compound SPH1-007-088 with the hydrophobic side chains of Gly83, Arg84, Lys105, Glu111, Asp131, Val132, Phe133, and Ile147. As for compound SPH1111-460 (Figure 5b), there are two hydrogen bonds formed with the SAM binding site. Oxygen O1 of SPH1111-460 creates a hydrogen bond with the side chain oxygen of Asp146, whereas oxygen O33 forms a hydrogen bond with the backbone nitrogen of Lys105. Meanwhile, compound 25SPH1-103-433 (Figure 5c) forms five hydrogen bonds between oxygen $\mathrm{O} 31$ and the carbonyl oxygen atom of Ser56, oxygen O32 and the side chain oxygen atom of Asp131, oxygen $\mathrm{O} 27$ and the side chain nitrogen atom of Arg84, oxygen O30 and the backbone nitrogen atom of Trp87, as well as the backbone nitrogen atom of Gly86. In addition, several hydrophobic interactions were found between compound 25SPH1-103-433 and Gly81, Cys82, Gly83, Thr104, Lys105, Phe133, and Ile147.

Dong et al. [24] suggested that four residues are significantly important ligand binding in the SAM binding pocket: Lys105, His110, Asp131, and Ile147. Three of the compounds we discovered create one or more interactions with the four important residues in the SAM binding site. Compound SPH1-007-088 binds to Lys105, Asp131, and Ile147. Compound SPH1-111-460 binds to Lys105, and compound 25SPH1-103-433 interacts with Lys105, Asp131, and Ile147. Our results indicate that these three compounds can block the SAM binding site by binding to its critical residues.

\section{Discussion}

Dengue and DHF remain pharmacologically neglected diseases [25] despite the high mortality rate, especially in developing countries where access to healthcare facilities is limited. Economic arguments have hampered the progress in discovering effective therapies for dengue [26]. The fact that dengue primarily affects poor communities prevents many pharmaceutical companies from making a serious effort to find the cure for this disease. Therefore, our research group has taken an initiative to be involved in the discovery of dengue chemotherapy by utilizing a limited laboratory setting similar to those commonly found in many developing countries.

We chose to perform a virtual screen of more than 5 million commercially available chemical compounds for two reasons. First, the cost of a screening campaign is sometimes prohibitive for laboratories in poor countries [27]. Therefore, virtual screening using computational methods may be performed to complement the "real" high-throughput screening (HTS) in less-funded laboratories. However, this approach has several disadvantages compared to HTS. For example, receptor flexibility is sometimes ignored in order to speed-up the computational screening. Second, the "drug universe" (including all the possible drug-like molecules) consists of $10^{62}$ molecules [28]. Thus, it is likely that potential molecules for dengue chemotherapy can be identified from the large universe of chemical compounds. To find potential chemotherapeutic compounds, the best strategy is not to synthesizing more compounds, but rather to use screening strategies to reduce the size of the molecular universe, as eloquently stated by Lahana in 1999: "when trying to find a needle in a haystack, the best strategy might not be to increase the size of the haystack" [28].

Performing virtual screening from a large database of chemical compounds requires access to multiprocessor computers, either in cluster or grid-based architectures. Research institutions in developed countries have launched a number of grid-based drug discovery efforts [29-31]; however, similar facilities often do not exist in developing countries. This fact motivated us to utilize two web-based drug-like compound databases (EDULISS and LIDAEUS) for the screening of commercially available chemical compounds as potential inhibitors of dengue NS5 MTase. The compounds screened from both databases were subsequently ranked based on their binding affinities to NS5 MTase using AutoDock Vina run on a single personal computer. The settings used in our experiments are suitable for most laboratories in developing countries with facilities that are inadequate for HTS and limited access to powerful multiprocessor computers.

In this work, we discovered several compounds that are potentially able to block the interaction between dengue NS5 MTase and its substrates, suggested that they may be able to stop the DV from replicating. The selection of potential compounds was based on their binding affinities. Compounds with stronger binding affinities for the RNA cap and SAM binding sites than that of SAH and RTP (NS5 MTase active ligands) were selected as potential inhibitors. In addition, the selected compounds must interact with residues that are catalytically important for the function of NS5 MTase. The mode of interaction between the compounds with the highest affinities and their binding sites was also discussed in detail.

\section{RNA cap site}

It has been suggested that nucleoside analogues are among the best NS5 MTase inhibitors. These compounds are expected to bind to the RNA binding site based on the fact that the strongest interaction between guanosine-5'-triphosphate (GTP, the natural substrate for NS5 MTase) and the RNA cap site is formed by $\pi-\pi$ stacking 
between Phe25 and the guanine moiety of GTP [14]. Ribavirin, a nucleoside analogue and a broad antiviral agent, has been shown to inhibit the replication of several DNA and RNA viruses [32], including the DV, although with weak activity $\left(\mathrm{EC}_{50}\right.$ of $\left.49 \mu \mathrm{g} / \mathrm{mL}\right)$ [33]. Ribavirin triphosphate (RTP) has also been shown to inhibit dengue NS5 MTase with low activity [34]. Figure 3a shows that RTP forms $\pi-\pi$ stacking between Phe25 and the pseudobase moiety of RTP. Other nucleoside analogues have also been synthesized as antiviral agents, such as 5-ethynyl-1- $\beta$-D-ribofuranosylimidazole-4-carboxyamide [35], and 7-deaza-2'-C-methyladenosine [36].

Our screening results show that none of the discovered compounds can be classified as nucleoside analogues. Nevertheless, the three compounds (SPH1-103-799, SPH1-101-102, and 28SPH1-115-917) show better binding to the RNA cap site than RTP, as indicated by their binding affinities. Our results are in agreement with other findings showing that molecules without nucleoside moieties may have biological activity against NS5 MTase. Aurintricarboxylic acid was determined to be a potential inhibitor at the RNA cap site of NS5 MTase by binding tightly to Lys61 [37], a residue necessary for correct 2'-O methylation of the RNA [15]. Interestingly, this compound binds to the two binding sites on NS5 MTase (RNA cap site and SAM binding site) with different $\mathrm{IC}_{50}$ values (2.3 and $127 \mu \mathrm{M}$, respectively) [37]. Podvinec et al. also suggested two non-nucleoside analogues that act as potential inhibitors of NS5 MTase by binding to the RNA cap site with $\mathrm{IC}_{50}$ values of 4.9 and $7.1 \mu \mathrm{M}$ [31].

\section{SAM binding site}

In contrast to the RNA cap site, the SAM binding site is rather closed and long. This has hindered the development of several SAM analogues as NS5 MTase inhibitors, presumably due to off-target activity of the compounds $[38,39]$. Efforts have been made to overcome this problem. For example, Lim et al. synthesized SAH derivatives that bind selectively to the SAM binding site at submicromolar concentrations. These compounds are thought to specifically bind to dengue NS5 MTase and do not interfere with related human enzymes [40].

It has been suggested that the guanosine moiety is important for promoting the activity of inhibitors that bind to the SAM binding site due to the strong hydrophobic contacts between the guanosine ring and Lys105 and Ile147 [24]. Nevertheless, Podvinec et al. reported that two compounds with no guanosine moiety bound to the SAM binding site $\left(\mathrm{IC}_{50}\right.$ of 4.4 and $\left.9.5 \mu \mathrm{M}\right)$ [31]. Our results confirm the findings by Podvinec et al. in that none of the compounds we discovered contain a guanosine moiety. All three compounds (SPH1-007-088, SPH1-111-460, and 25SPH1-103-433) bind to the SAM binding site by interacting with several residues important for MTase activity, such as Lys105, Asp131, and Ile147 [24].

Interestingly, the aromatic rings of compounds SPH1007-088 and 25SPH1-103-433 create hydrophobic contacts with Phe133 (Figure 5a and 5c). Phe133 was not identified as one of the important residues for MTase catalytic activity by Dong et al [24]. However, in their recent publication, Lim et al. [40] identified a new hydrophobic cavity that consists of residues Phe133, Ile147, Gly148, Glu149, Arg160, Arg163, Val164, and Leu182. Phe133 blocks the SAM binding site in its native conformation, and this residue flips away from the cavity by 100 degrees upon binding to larger substrates. Compounds SPH1-007088 and 25SPH1-103-433 bind to Phe133, suggesting that these compounds may restrain or prevent the hydrophobic cavity of the SAM binding site from opening.

\section{Conclusions}

We have discovered several potential inhibitory compounds of dengue virus NS5 MTase through virtual screening using structure-based and ligand-based methods. These compounds were predicted to bind to the SAM and RNA cap sites with higher affinities than SAH and RTP. These compounds are commercially available and can be easily purchased for further biological activity tests.

All of the compounds we discovered in this work bind to their respective binding sites by creating hydrogen bonds and hydrophobic interactions with important residues in the binding pockets. We performed a detailed analysis of the atomic interactions between each potential compound and residues inside the SAM binding site and RNA cap site to identify which residues interact with the compounds. We have shown that the interaction between all compounds with the SAM binding site and RNA cap site are facilitated by hydrogen bonds and hydrophobic contacts with residues that are vital for NS5 MTase catalytic function. Therefore, these compounds may be used as leads for developing an effective dengue drug.

Our virtual screening experiments were performed in a laboratory setting that is commonly found in many developing countries. We decided not to develop an expensive multiprocessor computer architecture. Rather we utilized two free, web-based drug-like chemical compound databases (LIDAEUS and EDULISS) and the freely available docking program AutoDock Vina. The new algorithm implemented in AutoDock Vina permits the high-throughput docking of thousands of compounds in a shorter time compared to the classical AutoDock program. Thus, it only requires a single personal computer. Similar experiments can be carried out in laboratories with limited funding and facilities. Therefore, we hope that more researchers in developing countries can be actively involved in the global effort to fight dengue infection. 


\section{Methods}

\section{Software and program}

PyMol (DeLano Scientific LLC, USA) and DS Visualizer (Accelrys, Inc., USA) were employed to visualize and modify the receptor and ligand structures. A molecular format conversion program, OpenBabelGUI, was used to prepare ligands for virtual screening and docking processes. The LIDAEUS (LIgand Discovery At Edinburgh UniverSity) [22] program was used to search for potential inhibitors based on a map of the NS5 MTase binding site by screening approximately 5 million commercially available chemical compounds. EDULISS (EDinburgh University LIgand Selection System) [23] is a chemical compound database that was used throughout this study to screen for potential inhibitors based on structural similarities with the known natural NS5 MTase substrates RTP and SAH. AutoDock Vina [41] was the primary docking program used in this work. The preparation of the MTase $p d b q t$ file and determination of the grid box size were carried out using AutoDock Tools version 1.5.4 (The Scripps Research Institute, La Jolla, USA). The PaDel-ADV program (Department of Pharmacy, National University of Singapore, Singapore) was utilized as the interface to perform molecular docking of the chemical compounds obtained from LIDAEUS and EDULISS using AutoDock Vina. Post-docking analyses were carried out using the Program of Energetic Analysis of Receptor Ligand System (PEARLS) [42] and LigPlot [43].

\section{Preparation of NS5 MTase structure}

The three-dimensional structure of NS5 MTase complexed with $S$-adenosyl-L-homocysteine $(\mathrm{SAH})$ and ribavirin (RTP) was retrieved from the Protein Data Bank [PDB:1R6A]. The NS5 MTase structure was prepared for structure-based virtual screening and molecular docking processes by removing all sulfate ions and water molecules. Ligands (SAH and RTP) were removed and saved as two separate $p d b$ files for further ligand-based virtual screening and control docking.

\section{Structure-based virtual screening}

The web-based program LIDAEUS (http://opus.bch.ed.ac. uk/lidaeus/index.php) [22] was utilized to search for potential inhibitors against NS5 MTase by matching the binding site map of the receptor against chemical compounds in the database. First, the NS5 MTase structure was uploaded into LIDAEUS followed by SAH and RTP separately. LIDAEUS generated an energy map and site points on the MTase structure based on the positions where SAH and RTP reside in their binding pockets (the SAM binding site and RNA cap binding site, respectively). Then, approximately 5 million compounds were screened, and the top 500 compounds were saved for further molecular docking.

\section{Ligand-based virtual screening}

Virtual screening based on the structure of NS5 MTase active ligands (SAH and RTP) was carried out using the web-based EDULISS program (http://eduliss.bch.ed.ac. $\mathrm{uk} /$ ) [23]. Two-dimensional structures of SAH and RTP were used to search for similar compounds in the EDULISS database from the most common chemical suppliers (ChemBridge, MayBridge, PubChem, Sigma-Aldrich, Salor, Fluka and Specs), covering approximately one million chemical compounds. The concept of the similarity search employed in EDULISS is based on Ultra Fast Shape Recognition with Atom Types (UFSRAT) [44]. The top 500 compounds with the highest structural similarity to SAH and RTP were used for further molecular docking analysis.

\section{Molecular docking}

Molecular docking was performed using AutoDock Vina [41]. Autodock Vina was used due to its accuracy and it speed, which is approximately two orders of magnitude faster than its predecessor, AutoDock 4 [45]. AutoDock Tools was utilized to prepare the input $p d b q t$ file for NS5 MTase and to set the size and the center of the grid box. Kollman charges and polar hydrogen atoms were added to the NS5 MTase structure. Because NS5 MTase contains two active sites, the grid box cavity size and center were set separately for both sites. The RNA cap site was set at $15.180 \times-51.931 \times 17.429$ in the dimensions of $x, y$ and $\mathrm{z}$ using $1.000 \AA$ spacing, while the SAM binding site was set at $18.002 \times-43.995 \times-1.503$ using $1.000 \AA$ spacing. AutoDock Vina requires the pdbqt input files of ligands to be prepared using AutoDock Tools. Due to the number of molecules to be docked (2000 compounds), the PaDel-ADV program was utilized to automate the docking process and act as an interface to perform the molecular docking of those compounds using AutoDock Vina. The predicted binding affinity $(\mathrm{kcal} / \mathrm{mol})$, which indicates how strongly a ligand binds to the receptor, is calculated based on the scoring function used in AutoDock Vina. A more negative binding affinity indicates stronger binding. The scoring function in AutoDock Vina is divided into two parts: i) a conformation-dependent part that can be seen as a sum of intramolecular and intermolecular contributions, including steric, hydrophobic, and hydrogen bonding interactions, and ii) a conformation-independent part that depends on the number of rotatable bonds between heavy atoms in the ligand. Each contribution (steric, hydrophobic, hydrogen bonding, and number of rotatable bonds) is given a different weight in the AutoDock Vina scoring function [41]. The validation of docking was carried out by redocking the NS5 MTase active ligands (SAH and RTP) into their binding sites. The Program of Energetic Analysis of 
Receptor Ligand System (PEARLS) and LigPlot programs were used to do post-docking analyses.

\section{List of abbreviations}

DV: dengue virus; GTP: guanosine-5'-triphosphate; MTase: methyltransferase; NS5: nonstructural protein 5; RTP: ribavirin triphosphate; SAH: S-adenosyl-Lhomocysteine; SAM: S-adenosyl-L-methionine.

\section{Acknowledgement}

The authors thank the Malaysian Ministry of Science, Technology and Innovation (MOSTI) for funding.

This article has been published as part of BMC Bioinformatics Volume 12 Supplement 13, 2011: Tenth International Conference on Bioinformatics First ISCB Asia Joint Conference 2011 (InCoB/ISCB-Asia 2011): Bioinformatics. The full contents of the supplement are available online at http://www. biomedcentral.com/1471-2105/12?issue=S13.

\section{Author details}

'Department of Chemistry, Faculty of Science, Universiti Putra Malaysia, 43400 UPM Serdang, Malaysia. ${ }^{2}$ Structural Biology Research Centre, Malaysia Genome Institute, UKM-MTDC Technology Centre, Universiti Kebangsaan Malaysia, 43600 UKM Bangi, Malaysia.

\section{Authors' contributions}

SVL conducted the experiments and drafted the manuscript. MBAR participated in the setting of computer resources and experimental design. BAT conceived of the study, participated in its design and coordination and helped to draft the manuscript. All authors read and approved the final manuscript.

\section{Competing interests}

The authors declare that they have no competing interests.

Published: 30 November 2011

\section{References}

1. Gubler DJ: Dengue and dengue hemorrhagic fever. Clin Microbiol Rev 1998, 11(3):480-496.

2. Ligon BL: Dengue fever and dengue hemorrhagic fever: a review of the history, transmission, treatment, and prevention. Semin Pediatr Infect Dis 2005, 16(1):60-65.

3. Gratz NG: Emerging and resurging vector-borne diseases. Annu Rev Entomol 1999, 44:51-75.

4. Halstead SB: Dengue. Lancet 2007, 370(9599):1644-1652.

5. Mackenzie JS, Gubler DJ, Petersen LR: Emerging flaviviruses: the spread and resurgence of Japanese encephalitis, West Nile and dengue viruses. Nat Med 2004, 10(12 Suppl):S98-109.

6. Perera R, Kuhn RJ: Structural proteomics of dengue virus. Curr Opin Microbiol 2008, 11(4):369-377.

7. Guo JT, Hayashi J, Seeger C: West Nile virus inhibits the signal transduction pathway of alpha interferon. J Virol 2005, 79(3):1343-1350.

8. Padmanabhan R, Mueller N, Reichert E, Yon C, Teramoto T, Kono Y, Takhampunya R, Ubol S, Pattabiraman N, Falgout B, et al: Multiple enzyme activities of flavivirus proteins. Novartis Found Symp 2006, 277:74-84, discussion 84-76, 251-253.

9. Podvinec M, Schwede T, Peitsch MC: Docking for neglected diseases as community efforts. In Computational Structural Biology: Methods and Applications. Singapore: World Scientific Publishing;Schwede T, Peitsch MC 2008:683-704.

10. Luzhkov VB, Selisko B, Nordqvist A, Peyrane F, Decroly E, Alvarez K, Karlen A, Canard B, Qvist J: Virtual screening and bioassay study of novel inhibitors for dengue virus mRNA cap (nucleoside-2'O)-methyltransferase. Bioorg Med Chem 2007, 15(24):7795-7802.

11. Cleaves GR, Dubin DT: Methylation status of intracellular dengue type 2 40 S RNA. Virology 1979, 96(1):159-165.

12. Ray D, Shah A, Tilgner M, Guo Y, Zhao Y, Dong H, Deas TS, Zhou Y, Li H, Shi PY: West Nile virus $5^{\prime}$-cap structure is formed by sequential guanine $\mathrm{N}-7$ and ribose 2'-O methylations by nonstructural protein 5. J Virol 2006, 80(17):8362-8370.
13. Egloff MP, Benarroch D, Selisko B, Romette JL, Canard B: An RNA cap (nucleoside-2'-O-)-methyltransferase in the flavivirus RNA polymerase NS5: crystal structure and functional characterization. Embo J 2002, 21(11):2757-2768.

14. Geiss BJ, Thompson AA, Andrews AJ, Sons RL, Gari HH, Keenan SM, Peersen OB: Analysis of flavivirus NS5 methyltransferase cap binding. J Mol Biol 2009, 385(5):1643-1654.

15. Zhou Y, Ray D, Zhao Y, Dong H, Ren S, Li Z, Guo Y, Bernard KA, Shi PY, Li H: Structure and function of flavivirus NS5 methyltransferase. J Virol 2007. 81(8):3891-3903

16. Courageot MP, Frenkiel MP, Dos Santos CD, Deubel V, Despres P: Alphaglucosidase inhibitors reduce dengue virus production by affecting the initial steps of virion morphogenesis in the endoplasmic reticulum. $J$ Virol 2000, 74(1):564-572.

17. Lim SP, Wen D, Yap TL, Yan CK, Lescar J, Vasudevan SG: A scintillation proximity assay for dengue virus NS5 2'-O-methyltransferase-kinetic and inhibition analyses. Antiviral Res 2008, 80(3):360-369.

18. Benarroch D, Egloff MP, Mulard L, Guerreiro C, Romette JL, Canard B: A structural basis for the inhibition of the NS5 dengue virus mRNA 2'-Omethyltransferase domain by ribavirin 5'-triphosphate. J Biol Chem 2004, 279(34):35638-35643

19. Morens DM: Antibody-dependent enhancement of infection and the pathogenesis of viral disease. Clin Infect Dis 1994, 19(3):500-512.

20. Kummerer BM, Rice CM: Mutations in the yellow fever virus nonstructural protein NS2A selectively block production of infectious particles. I Virol 2002, 76(10):4773-4784.

21. Stevens AJ, Gahan ME, Mahalingam S, Keller PA: The medicinal chemistry of dengue fever. J Med Chem 2009, 52(24):7911-7926.

22. Taylor P, Blackburn E, Sheng YG, Harding S, Hsin KY, Kan D, Shave S, Walkinshaw MD: Ligand discovery and virtual screening using the program LIDAEUS. Br J Pharmacol 2008, 153(Suppl 1):S55-67.

23. Hsin KY, Morgan HP, Shave SR, Hinton AC, Taylor P, Walkinshaw MD: EDULISS: a small-molecule database with data-mining and pharmacophore searching capabilities. Nucleic Acids Res 2011, 39(Database issue):D1042-1048.

24. Dong H, Chang DC, Xie X, Toh YX, Chung KY, Zou G, Lescar J, Lim SP, Shi PY: Biochemical and genetic characterization of dengue virus methyltransferase. Virology 2010, 405(2):568-578.

25. Pang T: Vaccines for the prevention of neglected diseases-dengue fever. Curr Opin Biotechnol 2003, 14(3):332-336.

26. Halstead SB, Deen J: The future of dengue vaccines. Lancet 2002, 360(9341):1243-1245.

27. Subramaniam S, Mehrotra M, Gupta D: Virtual high throughput screening (vHTS)-a perspective. Bioinformation 2008, 3(1):14-17.

28. Lahana R: How many leads from HTS? Drug Discov Today 1999, 4(10):447-448.

29. Chang MW, Lindstrom W, Olson AJ, Belew RK: Analysis of HIV wild-type and mutant structures via in silico docking against diverse ligand libraries. J Chem Inf Model 2007, 47(3):1258-1262.

30. Kasam V, Zimmermann M, Maass A, Schwichtenberg H, Wolf A, Jacq N, Breton V, Hofmann-Apitius M: Design of new plasmepsin inhibitors: a virtual high throughput screening approach on the EGEE grid. J Chem Inf Model 2007, 47(5):1818-1828.

31. Podvinec M, Lim SP, Schmidt T, Scarsi M, Wen D, Sonntag LS, Sanschagrin P, Shenkin PS, Schwede T: Novel inhibitors of dengue virus methyltransferase: discovery by in vitro-driven virtual screening on a desktop computer grid. J Med Chem 2010, 53(4):1483-1495.

32. Sidwell RW, Huffman JH, Khare GP, Allen LB, Witkowski JT, Robins RK: Broad-spectrum antiviral activity of Virazole: 1-beta-D-ribofuranosyl1,2,4-triazole-3-carboxamide. Science 1972, 177(50):705-706.

33. Gabrielsen B, Phelan MJ, Barthel-Rosa L, See C, Huggins JW, Kefauver DF, Monath TP, Ussery MA, Chmurny GN, Schubert EM, et al: Synthesis and antiviral evaluation of $\mathrm{N}$-carboxamidine-substituted analogues of 1-betaD-ribofuranosyl-1,2,4-triazole-3-carboxamidine hydrochloride. J Med Chem 1992, 35(17):3231-3238.

34. Crance JM, Scaramozzino N, Jouan A, Garin D: Interferon, ribavirin, 6azauridine and glycyrrhizin: antiviral compounds active against pathogenic flaviviruses. Antiviral Res 2003, 58(1):73-79.

35. Koff WC, Pratt RD, Elm JL Jr., Venkateshan CN, Halstead SB: Treatment of intracranial dengue virus infections in mice with a lipophilic derivative of ribavirin. Antimicrob Agents Chemother 1983, 24(1):134-136. 
36. Olsen DB, Eldrup AB, Bartholomew L, Bhat B, Bosserman MR, Ceccacci A, Colwell LF, Fay JF, Flores OA, Getty KL, et al: A 7-deaza-adenosine analog is a potent and selective inhibitor of hepatitis $C$ virus replication with excellent pharmacokinetic properties. Antimicrob Agents Chemother 2004 48(10):3944-3953.

37. Milani M, Mastrangelo E, Bollati M, Selisko B, Decroly E, Bouvet M, Canard B, Bolognesi M: Flaviviral methyltransferase/RNA interaction: structural basis for enzyme inhibition. Antiviral Res 2009, 83(1):28-34.

38. Vedel M, Lawrence F, Robert-Gero M, Lederer $\mathrm{E}$ : The antifungal antibiotic sinefungin as a very active inhibitor of methyltransferases and of the transformation of chick embryo fibroblasts by Rous sarcoma virus. Biochem Biophys Res Commun 1978, 85(1):371-376.

39. Yebra MJ, Sanchez J, Martin CG, Hardisson C, Barbes C: The effect of sinefungin and synthetic analogues on RNA and DNA methyltransferases from Streptomyces. J Antibiot (Tokyo) 1991, 44(10):1141-1147.

40. Lim SP, Sonntag LS, Noble C, Nilar SH, Ng RH, Zou G, Monaghan P, Chung KY, Dong H, Liu B, et al: Small molecule inhibitors that selectively block dengue virus methyltransferase. J Biol Chem 2011, 286(8):6233-6240.

41. Trott O, Olson AJ: AutoDock Vina: improving the speed and accuracy of docking with a new scoring function, efficient optimization, and multithreading. J Comput Chem 2010, 31(2):455-461.

42. Han LY, Lin HH, Li ZR, Zheng CJ, Cao ZW, Xie B, Chen YZ: PEARLS: program for energetic analysis of receptor-ligand system. J Chem Inf Model 2006, 46(1):445-450.

43. Wallace AC, Laskowski RA, Thornton JM: LIGPLOT: a program to generate schematic diagrams of protein-ligand interactions. Protein Eng 1995, 8(2):127-134.

44. Shave SR: The development of high performances structure and ligand based virtual screening techniques. PhD Thesis Edinburgh: University of Edinburgh; 2009.

45. Morris GM, Huey R, Olson AJ: Using AutoDock for ligand-receptor docking. Curr Protoc Bioinformatics 2008, Chapter 8(Unit 8):14.

doi:10.1186/1471-2105-12-S13-S24

Cite this article as: Lim et al:: Structure-based and ligand-based virtual screening of novel methyltransferase inhibitors of the dengue virus. BMC Bioinformatics 2011 12(Suppl 13):S24.

\section{Submit your next manuscript to BioMed Central and take full advantage of:}

- Convenient online submission

- Thorough peer review

- No space constraints or color figure charges

- Immediate publication on acceptance

- Inclusion in PubMed, CAS, Scopus and Google Scholar

- Research which is freely available for redistribution

Submit your manuscript at www.biomedcentral.com/submit
Ciomed Central 